\title{
EGRET Spectral Index and the Low-Energy Peak Position in the Spectral Energy Distribution of EGRET-Detected Blazars
}

\author{
Y. C. $\operatorname{Lin}^{1,2}$, D. L. Bertsch ${ }^{3}$, S. D. Bloom ${ }^{4}$, J. A. Esposito ${ }^{5}$, R. C. Hartman ${ }^{3}$, S. D. Hunter ${ }^{3}$, \\ G. Kanbach ${ }^{6}$, D. A. Kniffen ${ }^{7}$, H. A. Mayer-Hasselwander ${ }^{6}$, P. F. Michelson ${ }^{1}$, \\ R. Mukherjee ${ }^{8}$, A. M. Mücke ${ }^{9}$, P. L. Nolan ${ }^{1}$, M. Pohl ${ }^{10}$, O. Reimer ${ }^{6}$, E. J. Schneid ${ }^{11}$, \\ D. J. Thompson ${ }^{3}$, W. F. Tompkins ${ }^{1}$
}

Received —

\footnotetext{
${ }^{1}$ W. W. Hansen Experimental Physics Laboratory, Stanford University, Stanford CA 94305 USA

${ }^{2}$ lin@egret0.stanford.edu

${ }^{3}$ Code 661 , Laboratory for High Energy Astrophysics, NASA Goddard Space Flight Center, Greenbelt, MD 20771 USA

${ }^{4}$ IPAC, Jet Propulsion Laboratory, California Institute of Technology, Pasadena, CA 91125 USA

${ }^{5}$ Research and Data Systems Corporation, 7501 Forbes Blvd., Suite 104, Seabrook, MD 20706 USA

${ }^{6}$ Max-Planck-Institut für Extraterrestrische Physik, Giessenbachstr D-85748 Garching Germany

${ }^{7}$ Department of Physics, Hampden-Sydney College, Hampden-Sydney, VA 23943 USA

${ }^{8}$ Barnard College and Columbia University, New York, NY 10027 USA

${ }^{9}$ Dept. of Phys. and Mathematical Phys., Univ. of Adelaide, Adelaide, SA 5005 Australia

${ }^{10}$ Institut für Theoretische Physik 4, Ruhr-Universität Bochum, 44780 Bochum Germany

${ }^{11}$ Northrop-Grumman Aerospace Corporation, Mail Stop A01-26, Bethpage, NY 11714 USA
} 


\begin{abstract}
In current theoretical models of the blazar subclass of active galaxies, the broadband emission consists of two components: a low-frequency synchrotron component with a peak in the IR to X-ray band, and a high-frequency inverse Compton component with a peak in the gamma-ray band. In such models, the gamma-ray spectral index should be correlated with the location of the low-energy peak, with flatter gamma-ray spectra expected for blazars with synchrotron peaks at higher photon energies and vice versa. Using the EGRET-detected blazars as a sample, we examine this correlation and possible uncertainties in its construction.
\end{abstract}

Subject headings: gamma rays: general; galaxies: active 


\section{Introduction}

It is now generally believed in a class of theoretical models (see e.g. Ulrich, Maraschi, \& Urry 1997 for a review) that the broadband spectrum of a blazar consists of two distinctive components: (a) a low-energy component which is the result of synchrotron radiation of a beam of relativistic particles, and which peaks, in the spectral energy distribution (SED) plot, in the IR to soft X-ray region; (b) a high-energy component which is the result of

inverse Compton scattering of the same beam of relativistic particles on some ambient field of soft photons, and which peaks in SED in the MeV-GeV-TeV region. These models are well known for their attempt to explain the most salient features of the broadband spectra of blazars from radio energies all the way to the TeV energies, an energy span of more than 20 orders of magnitude.

In this paper we examine an important prediction of this class of theoretical models with EGRET data. The two broad peaks in SED of a blazar as described in such models, being the products of the same beam of relativistic particles, should be closely related to each other. Since the high-energy peaks in SED of various blazars pass through the EGRET energy range from $\sim 30 \mathrm{MeV}$ to $20 \mathrm{GeV}$, the spectral shapes of the EGRET-detected blazars in the EGRET energy range should change systematically with respect to the positions of the low-energy SED peaks in different objects. This prediction of these currently investigated theoretical models can be tested with EGRET data.

A brief and preliminary result of a study of this kind with EGRET data, based on the Second EGRET Catalog and its Supplement (Thompson et al. 1995, Thompson et al. 1996), has already been sent for publication in the Proceedings of the BL Lac Phenomenon Meeting of 1998 in Turku, Finland (Lin et al. 1998). In the present paper, we expand the scope of the previous study with additional information taken from the recently published Third EGRET Catalog (Hartman et al. 1999) and other publications to examine again in 
more details the question of the possible correlation between EGRET spectral shapes and low-energy SED peak positions for the blazars that have been detected by EGRET.

\section{The Data}

We select 27 EGRET-detected blazars (Fichtel et al. 1994, Thompson et al. 1995, Thompson et al. 1996, Hartman et al. 1999). These are the ones for which the SED can be found in the published data at least to the extent that the low-energy peak positions can be determined, and for which the EGRET photon spectra can also be determined. Four of the sources in this sample are traditionally regarded as X-ray-selected BL Lac objects (XBL, see Ciliegi et al. 1995). Recently these objects have been reclassified as high-energy peaked BL Lac objects (HBL, see Ulrich, Maraschi, \& Urry 1997). Another eleven of these sources are usually regarded as radio-selected BL Lac objects (RBL, see Ciliegi et al. 1995), or reclassified as low-energy peaked BL Lac objects (LBL, see e.g. Ulrich, Maraschi, \& Urry 1997). The other twelve sources in the sample belong to what are generally referred to as flat-spectrum radio quasars (FSRQ). For two of the four XBL (HBL), Mrk 501 and PKS 2005-489, the EGRET detections are somewhat weak but still fairly certain (Kataoka et al. 1999, Sreekumar et al. 1999, Lin et al. 1997). Three of the four XBL have been detected at $\mathrm{TeV}$ energies (see e.g. Krennrich et al. 1999 or Macomb et al. 1995 for Mrk 421, Kataoka et al. 1999 or Kennrich et al. 1999 for Mrk 501, and Chadwick et al. 1999 for PKS 2155-304), while a good TeV flux upper limit exists for the fourth one (PKS 2005-489, Roberts et al. 1998). Thus the SED of these four XBL (HBL) can be constructed well into the $\mathrm{TeV}$ energies with the high-energy peaks

clearly seen. Furthermore, five of the EGRET-detected FSRQ have also been detected by OSSE and COMPTEL in the 0.05 to $15 \mathrm{MeV}$ energy range. Combined spectra have been determined for these five sources through the OSSE/COMPTEL/EGRET energy ranges 
(3C 273, 3C 279, CTA 102, PKS 0528+134, and 3C 454.3, see McNaron-Brown et al. 1995). These five sources are all included in the sample here. The high-energy peaks of these five sources can be constructed in the $\mathrm{MeV}$ to $\mathrm{GeV}$ energy range with strict simultaneous data (McNaron-Brown et al. 1995). These peak positions are visible as spectral break points between the OSSE/COMPTEL data and the EGRET data.

This sample of 27 EGRET-detected blazars are listed in Table 1 together with EGRET fluxes, EGRET spectral indices, and the low-energy SED peak frequencies. The EGRET fluxes and spectral indices are taken from the recent Third EGRET Catalog (Hartman et al. 1999) unless noted otherwise. Most of the sources in the EGRET catalogs carry multiple flux values. The values quoted here in Table 1 are the first entries in the Third EGRET Catalog upon which the source positions and the source identifications are determined. The EGRET spectral indices in the Third EGRET Catalog are those determined for the sum data of Cycle 1 through Cycle 4 (1991 April 22 to 1995 October 4). There are some evidence that the EGRET spectra of some blazars tend to become harder at higher flux levels (Mukherjee et al. 1997). But the variations are small and only become apparent for bright EGRET sources when the spectral indices can be determined with high degrees of accuracy. So the EGRET spectral indices listed in the Third EGRET Catalog and quoted here in Table 1, though calculated only as average values over long period of time, are good representation of the actual spectral shapes. The low-energy SED peak frequencies in Table 1 are taken from published data. References for these information are given in the footnotes below the table. On the average, the peak positions can be determined from the published data to an accuracy of about \pm 0.2 in the scale of $\log _{10}$ (frequency). Out of the 27 sources studied here, four are found to have enough data to show the low-energy SED peak frequency at different epochs: PKS 0235+164, Mrk 421, 3C 279, and BL Lacertae (see references cited in Table 1). The ranges of the peak frequencies of these four sources are 0.5 for PKS $0235+164,1.0$ for Mrk 421, 0.3 for 3C 279, and 0.2 for BL Lacertae, in 
the scale of $\log _{10}$ (frequency). These variations in the low-energy SED peak frequencies are small compared with the full frequency range of all blazars studied here. For these four sources, we enter the peak frequencies corresponding to quieter times in Table 1, as these are the situations where more abundant data are available. Finally some special features of the individual sources are included as remarks in the last column of Table 1. This source sample is not meant to be a complete one. We just try to construct a sample size that is sufficiently large to draw certain statistical conclusions.

\section{The Analysis}

To examine the correlation between the low-energy peak and the high-energy peak in the SED of a blazar, we should ideally try to match these two peaks over broad energy ranges that cover substantial segments of the spectrum. But this is not feasible at present with existing data. Only a handful of sources have detailed measurements of the spectra from radio energies to $\mathrm{TeV}$ energies. Conclusions drawn from these few sources are likely to be biased in some way and not generally applicable to blazars as a class. Most of the blazars in the literature have good measurements on their broadband spectra only around the low-energy SED peaks. For the EGRET-detected blazars in general, there are no existing data to show where the high-energy peak frequencies are located except for the few sources that are either the XBL mentioned above or the ones that have also been detected by OSSE and COMPTEL (McNaron-Brown et al. 1995), also mentioned above. To examine the correlation between the two SED peaks, we need to rely on some specific properties of the broadband spectra in the two energy regions.

For the low-energy peaks in SED, it is natural to examine the peak frequencies as these are the prominent spectral features that can be determined fairly accurately from published data. Then in the EGRET energy range we examine the spectral shapes, or more 
specifically the spectral indices to see if they systematically change with the low-energy peak frequencies.

In addition to examination of the model prediction on the correlation between the low-energy SED peak frequency and the EGRET spectral index, which involves only experimental data as described above, we can also compare the observed EGRET spectral indices with the calculated spectral indices from these theoretical models of blazars currently under investigation (see e.g. Ulrich, Maraschi, \& Urry 1997) in the EGRET energy region. We take the illustrative theoretical curves plotted in Figure 12 of Fossati et al. (1999) and determine the slopes of these curves at $100 \mathrm{MeV}$ as a function of the low-energy SED peak frequencies in the figure. We then compare these theoretical slopes with EGRET spectral indices of the sources listed in Table 1 as functions of the low-energy peak frequencies.

In Figure 1 we plot the EGRET spectral index $\gamma-2\left(\mathrm{f} \sim \mathrm{E}^{-\gamma}\right)$ vs. the low-energy SED peak frequency in $\log _{10}$ scale for the sample of 27 EGRET-detected blazars examined in this paper. The source designations, their low-energy SED peak frequencies, and the corresponding EGRET spectral indices are all listed in Table 1. The value $\gamma-2$ corresponds to the spectral index in an SED plot. The five FSRQ that are also detected by OSSE/COMPTEL are indicated separately in the figure. In Figure 1 we also plot the theoretical prediction of the spectral slope at $100 \mathrm{MeV}$ as a function of the low-energy SED peak frequency as described in the paragraph above. Some of the graph points in Figure 1 are slightly shifted in their abscissae to avoid graph congestion. The theoretically calculated spectral slopes at $100 \mathrm{MeV}$ are connected with dotted lines.

As one can see in Figure 1, the EGRET spectral indices do not vary systematically with respect to the low-energy SED peak frequencies. The four XBL (HBL) may form the only exception to this general situation. The theoretical models seem to work well for the four EGRET-detected XBL. But when the low-energy SED peak frequency moves toward 
lower values, the agreement between the theoretical predition and the EGRET data ceases to exist. The EGRET spectral indices do not form a pattern that may resemble the upturn of the theoretical curve toward smaller low-energy SED peak frequencies. One cannot find a trend of other kind either in the EGRET spectral indices in Figure 1. The error margins in the EGRET spectral indices cannot accommodate the discrepancy between the theoretical prediction and the EGRET data. Many of the EGRET spectral indices have been determined with an accuracy better than $\sim \pm 0.15$.

We may also add that for the four EGRET-detected XBL (HBL), the EGRET spectral indices $\gamma\left(\mathrm{f} \sim \mathrm{E}^{-\gamma}\right)$ all fall within the low range between 1.5 and 1.7 while the low-energy SED peak frequencies all fall within the high range between $10^{16}$ and $10^{17} \mathrm{~Hz}$. This fact is consistent with the results of the theoretical model fits (see e.g. Fossati et al. 1999). But when it comes to the individual spectral and peak frequency values, there is no correlation between the EGRET spectral indices and the low-energy SED peak frequencies for these four XBL either. However we must point out that the error margins in the EGRET spectral indices are large for these four XBL and the lack of correlation found here does not carry much weight. But for the five EGRET-detected FSRQ that have also been detected by OSSE/COMPTEL, and as such the high-energy SED peak frequencies can also be determined with the combined OSSE/COMPTEL/EGRET data as the spectral break points in the $0.015 \mathrm{MeV}$ to several GeV energy range (see Figure 2 in McNaron-Brown et al. 1995), these five sources do not show correlation between the low-energy SED peak frequencies and the high-energy SED peak frequencies. The blazars that can be detected by OSSE or COMPTEL are likely to be those with steep EGRET spectra because then the EGRET spectra will extend high into the COMPTEL/OSSE energy regions. We may expect to see better agreement between theory and data for these five sources alone where the EGRET spectral indices become high. But in Figure 1, these five sources do not follow the theoretical curve either. 


\section{Discussion}

Many of the EGRET-detected blazars suffer from poor statistical accuracy because of limited photon counts; in those cases, the spectral indices are very poorly determined. However, in Figure 1 it is apparent that the greatest discrepancy between the data and the theoretical prediction is at the lowest synchrotron-peak frequencies, where most of the best-determined EGRET indices are found. In the unified blazar scenarios it might be expected that the objects with synchrotron-peak frequencies below $10^{13} \mathrm{~Hz}$ are all FSRQ's; however, note that all of these are far below the (extrapolation of the) theoretical curve. The typical differences between the observed and predicted spectral indices are $\sim 0.8$, whereas the typical errors in those EGRET spectral indices are $\sim 0.15$; thus the discrepancies are clearly not due to statistical limitations. The EGRET-detected FSRQ's clearly have much harder spectra than the theory predicts.

The RBL's also do not agree well with the theoretical curve, although the cluster of points extends both above and below the theoretical curve. In this case the discrepancy appears as a broader distribution around the theoretical curve than would be expected from the errors in the EGRET indices. For example, four of the eleven points are more than $2 \sigma$ away from the theoretical curve, whereas statistically no more than one would be expected.

It is well-known that the low-energy SED peak frequencies of blazars may vary with flux levels. Since most of the SED spectra studies in this paper were constructed with noncontemporaneous data, one would have to consider the possibility that the lack of correlation between the low-energy SED peak frequency and the EGRET spectral index in Figure 1 could have been caused by the shift of the low-energy SED peak positions in different epochs. We are fairly certain that the EGRET spectra do not change appreciably with flux (Mukherjee et al. 1997). As for the shift of the low-energy SED peak positions, the well-studied sources like those mentioned at the end of Section 2 indicate that the 
amount of changes are no bigger that $\sim 1.0$ in the scale of $\log _{10}$ (frequency), or about one order of magnitude in frequency. But if we want to bring the FSRQ or RBL with hard EGRET spectra in Figure 1 to agree with the theoretical curve, we would have to shift their low-energy SED peak frequencies by at least three orders of magnitude. Such large change in position of the low-energy SED peak has never been observed, at least for the majority of the blazars under study in the literature.

Another possible inconsistency between the theoretical models and the EGRET data may exist when the spectral shapes of all EGRET blazars are viewed together, not restricted to the 27 sources listed in Table 1 . The currently studied theoretical blazar models require that some of the EGRET spectra should show clear spectral breaks when the high-energy SED peaks pass through the EGRET energy range, much like the spectral breaks of the five sources detected by all of OSSE, COMPTEL, and EGRET, with spectral breaks in SED clearly seen between the OSSE/COMPTEL data and the EGRET data (McNaron-Brown et al. 1995). But in all of the spectral fits that have been carried out by the EGRET Team (Fichtel et al. 1994, Thompson et al. 1995, Thompson et al. 1996, Hartman et al. 1999, and references therein), it has never been found necessary to introduce spectral breaks or spectral cutoffs in order to properly fit the EGRET data. It is of course entirely possible that some of the EGRET blazar spectra will eventually prove to be more complicated than single power laws when the measurements become sufficiently accurate. In fact, even with the existing EGRET data, Reimer et al. (1999) and Bertsch et al. (1999) are currently carrying out studies to see if functional forms more complicated than single power laws will provide better fit for some of the EGRET blazars. But we must point out that in the EGRET energy range, spectral breaks as conspicuous as those displayed in McNaron-Brown et al. (1995) or spectral cutoffs well below the GeV energy range can be easily recognized in the EGRET data. Thus if a theoretical model requires that some of the EGRET sources should possess spectral breaks or spectral cutoffs beyond what the statistical uncertainties 
in the EGRET data can accomodate, then the theoretical model will be inconsistent with EGRET data.

The EGRET team gratefully acknowledges support from the following:

Bundesministerium für Forschung und Technologie, Grant 50 QV 9095 (MPE authors); NASA Grant NAG5-1742 (HSC); NASA Grant NAG5-1605 (SU); and NASA Contract NAS5-31210 (GAC). 
Table 1. EGRET-Detected Blazars Examined in This Study.

\begin{tabular}{|c|c|c|c|c|c|}
\hline \multirow[t]{3}{*}{ Object } & Other & EGRET Flux & EGRET & Low-E Peak & \multirow[t]{3}{*}{ Remark } \\
\hline & \multirow[t]{2}{*}{ Name } & $\mathrm{E}>100 \mathrm{MeV}$ & Spectral & $\log _{10}\left(f_{\text {peak }}\right)$ & \\
\hline & & $10^{-7} \mathrm{~cm}^{-2} \mathrm{~s}^{-1}$ & Index $\gamma$ & $f_{\text {peak }}$ in $\mathrm{Hz}$ & \\
\hline $0208-512$ & & $8.55 \pm 0.45$ & $1.99 \pm 0.05$ & $12.0^{(1)}$ & FSRQ \\
\hline $0219+428$ & $3 \mathrm{C} 66 \mathrm{~A}$ & $1.87 \pm 0.29$ & $2.01 \pm 0.14$ & $13.0^{(2)}$ & RBL \\
\hline $0235+164$ & & $6.51 \pm 0.88$ & $1.85 \pm 0.12$ & $13.0^{(1)}$ & $\mathrm{RBL}$ \\
\hline $0420-014$ & & $5.02 \pm 1.04$ & $2.44 \pm 0.19$ & $12.3^{(1)}$ & FSRQ \\
\hline $0454-234$ & PKS & $1.47 \pm 0.42$ & $3.14 \pm 0.47$ & $13.5^{(2)}$ & FSRQ \\
\hline $0458-020$ & & $1.12 \pm 0.23$ & $2.45 \pm 0.27$ & $13.0^{(2)}$ & FSRQ \\
\hline $0521-365$ & & $3.19 \pm 0.72$ & $2.63 \pm 0.42$ & $13.5^{(2)}$ & RBL \\
\hline \multirow[t]{2}{*}{$0528+134$} & PKS & $9.35 \pm 0.36$ & $2.46 \pm 0.04$ & $12.0^{(1)}$ & FSRQ \\
\hline & & & & & OSSE/COMPTEL \\
\hline $0537-441$ & & $2.53 \pm 0.31$ & $2.41 \pm 0.12$ & $13.5^{(1)}$ & RBL \\
\hline $0716+714$ & & $1.78 \pm 0.20$ & $2.19 \pm 0.11$ & $13.8^{(3)}$ & RBL \\
\hline $0735+178$ & & $1.64 \pm 0.33$ & $2.60 \pm 0.28$ & $13.7^{(2)}$ & RBL \\
\hline $0829+046$ & & $1.68 \pm 0.51$ & $2.47 \pm 0.40$ & $13.6^{(2)}$ & RBL \\
\hline $0851+202$ & OJ +287 & $1.06 \pm 0.30$ & $2.03 \pm 0.35$ & $13.0^{(2)}$ & $\mathrm{RBL}$ \\
\hline $0954+658$ & & $1.54 \pm 0.30$ & $2.08 \pm 0.24$ & $13.6^{(2)}$ & $\mathrm{RBL}$ \\
\hline \multirow[t]{2}{*}{$1101+384$} & Mrk 421 & $1.39 \pm 0.18$ & $1.57 \pm 0.15$ & $16.0^{(4)}$ & $\mathrm{XBL}$ \\
\hline & & & & & TeV Detection \\
\hline $1156+295$ & $4 \mathrm{C}+29.45$ & $5.09 \pm 1.19$ & $1.98 \pm 0.22$ & $13.0^{(2)}$ & FSRQ \\
\hline $1219+285$ & W Comae & $1.15 \pm 0.18$ & $1.73 \pm 0.18$ & $13.5^{(1)}$ & RBL \\
\hline $1226+023$ & 3C 273 & $1.54 \pm 0.18$ & $2.58 \pm 0.09$ & $13.5^{(1)}$ & FSRQ \\
\hline & & & & & OSSE/COMPTEL \\
\hline
\end{tabular}


Table 1 - Continued

\begin{tabular}{|c|c|c|c|c|c|}
\hline Object & $\begin{array}{l}\text { Other } \\
\text { Name }\end{array}$ & $\begin{array}{l}\text { EGRET Flux } \\
\mathrm{E}>100 \mathrm{MeV} \\
10^{-7} \mathrm{~cm}^{-2} \mathrm{~s}^{-1}\end{array}$ & $\begin{array}{l}\text { EGRET } \\
\text { Spectral } \\
\text { Index } \gamma\end{array}$ & $\begin{array}{l}\text { Low-E Peak } \\
\log _{10}\left(f_{\text {peak }}\right) \\
f_{\text {peak }} \text { in } \mathrm{Hz}\end{array}$ & Remark \\
\hline $1253-055$ & 3C 279 & $17.97 \pm 0.67$ & $1.96 \pm 0.04$ & $12.6^{(5)}$ & $\begin{array}{c}\text { FSRQ } \\
\text { OSSE/COMPTEL }\end{array}$ \\
\hline $1510-089$ & & $1.80 \pm 0.38$ & $2.47 \pm 0.21$ & $13.0^{(2)}$ & FSRQ \\
\hline $1633+382$ & $4 \mathrm{C}+38.41$ & $10.75 \pm 0.96$ & $2.15 \pm 0.09$ & $12.3^{(1)}$ & FSRQ \\
\hline $1652+398$ & Mrk 501 & $3.20 \pm 1.30^{(6)}$ & $1.60 \pm 0.50^{(6)}$ & $16.7^{(6)}$ & $\begin{array}{c}\text { XBL } \\
\text { TeV Detection }\end{array}$ \\
\hline $2005-489$ & PKS & $1.31 \pm 0.46^{(7)}$ & $1.52 \pm 0.24^{(7)}$ & $16.4^{(8)}$ & $\begin{array}{c}\text { XBL } \\
\text { TeV Upper Limit }\end{array}$ \\
\hline $2155-304$ & PKS & $3.04 \pm 0.77$ & $1.71 \pm 0.24^{(9)}$ & $17.0^{(10)}$ & $\begin{array}{c}\mathrm{XBL} \\
\text { TeV Detection }\end{array}$ \\
\hline $2200+420$ & BL Lacertae & $3.99 \pm 1.16$ & $2.60 \pm 0.28$ & $14.0^{(11)}$ & RBL \\
\hline $2230+114$ & CTA 102 & $1.92 \pm 0.28$ & $2.45 \pm 0.14$ & $12.1^{(2)}$ & $\begin{array}{c}\text { FSRQ } \\
\text { OSSE/COMPTEL }\end{array}$ \\
\hline $2251+158$ & 3C 454.3 & $5.37 \pm 0.40$ & $2.21 \pm 0.06$ & $12.8^{(1)}$ & $\begin{array}{c}\text { FSRQ } \\
\text { OSSE/COMPTEL }\end{array}$ \\
\hline
\end{tabular}

References. - (1) von Montigny et al. 1995; (2) Impey \& Neugebauer 1988; (3) Ghisellini et al. 1997; (4) Macomb et al. 1995; (5) Wehrle et al. 1998; (6) Kataoka et al. 1999; (7) Lin et al. 1997; (8) Sambruna et al. 1995; (9) Vestrand et al. 1995; (10) Chadwick et al. 1999; (11) Catanese et al. 1997 


\section{REFERENCES}

Bertsch, D. L., et al. 1999, in preparation

Catanese, M., et al. 1997, ApJ, 480, 562

Chadwick, P. M., et al. 1999, ApJ, in press

Ciliegi, P., Bassani, L., \& Caroli, E. 1995, ApJ, 439, 80

Fichtel, C. E., et al. 1994, ApJ, 438, 80

Fossati, G., et al. 1999, MNRAS, in press

Ghisellini, G., et al. 1997, A\&A, 327, 61

Hartman, R. C., et al. 1999, ApJS, July issue, in press

Impey, C. D., \& Neugebauer, G. 1988, AJ, 95, 304

Kataoka, J., et al. 1999, ApJ, 514, in press

Krennrich, F., et al. 1998, ApJ, 511, 149

Lin, Y. C., et al. 1997, Proc. Fourth Compton Symposium, ed. C. D. Dermer, M. S. Strickman, \& J. D. Kurfess, AIP Conf. Proc. 410, p. 1371

Lin, Y. C., et al. 1998, Proc. BL Lac Phenomenon, ed. L. Takalo, in press

Macomb, D. J., et al. 1995, ApJ, 449, L99

McNaron-Brown, K., et al. 1995, ApJ, 451, 575

von Montigny, C., et al. 1995, ApJ, 440, 525

Mukherjee, R., et al. 1997, ApJ, 490, 116 
Reimer, O., et al. 1999, in preparation

Roberts, M. D., et al. 1998, A\&A, 337, 25

Sambruna, R. M., et al. 1995, ApJ, 449, 567

Sreekumar, P., et al. 1999, ApJ, to be submitted

Thompson, D. J., et al. 1995, ApJS, 101, 259

Thompson, D. J., et al. 1996, ApJS, 107, 227

Ulrich, M. H., Maraschi, M., \& Urry, C. M. 1997, Ann. Rev. Astro. Astrophys., 35, 445

Vestrand, W. T., et al. 1995, ApJ, 454, L93

Wehrle, A. E., et al. 1998, ApJ, 497, 178 
Fig. 1.- EGRET photon spectral index $\gamma-2$ vs. $\log _{10}$ (low-energy peak frequency in SED) for EGRET-detected blazars. 


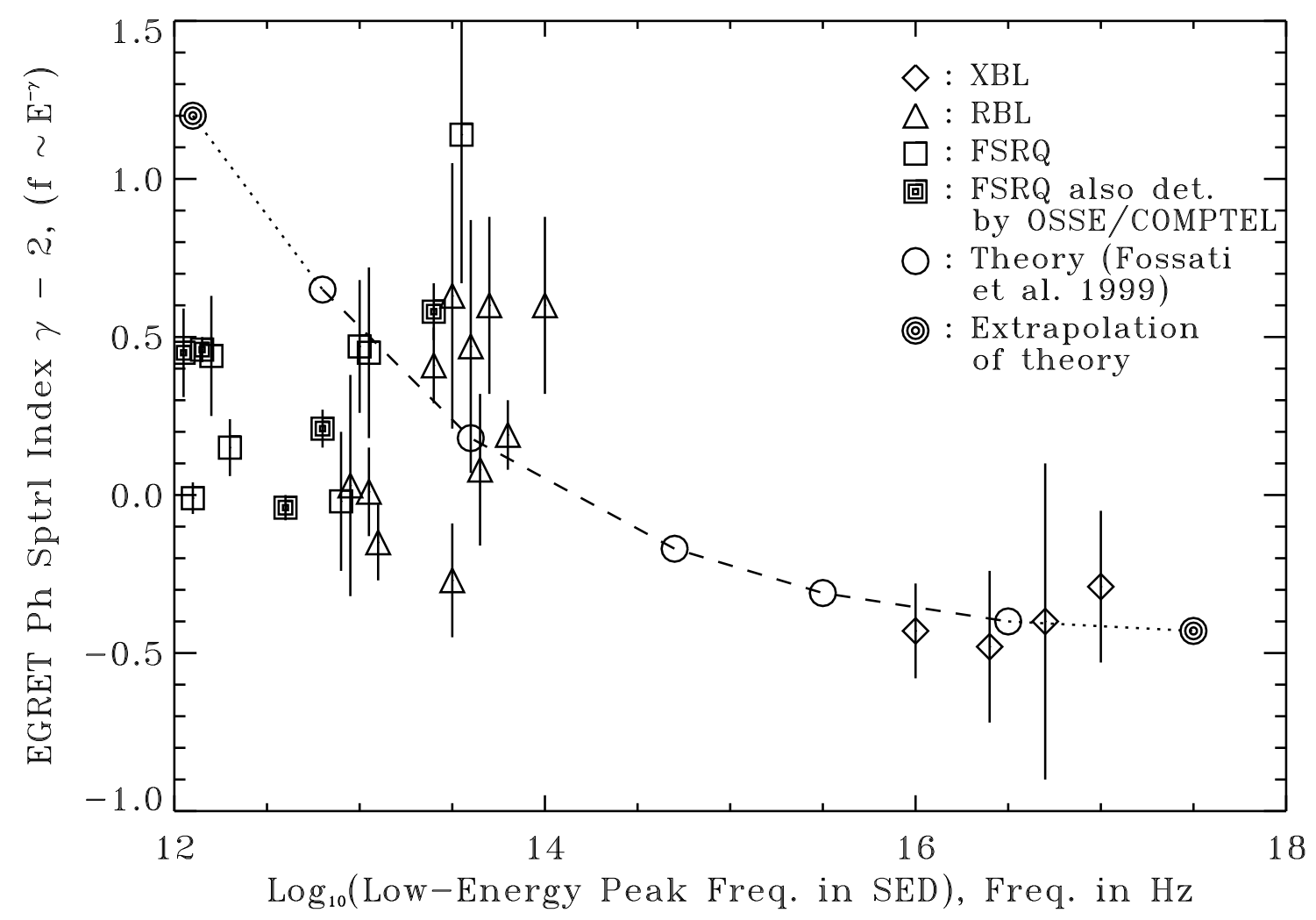

